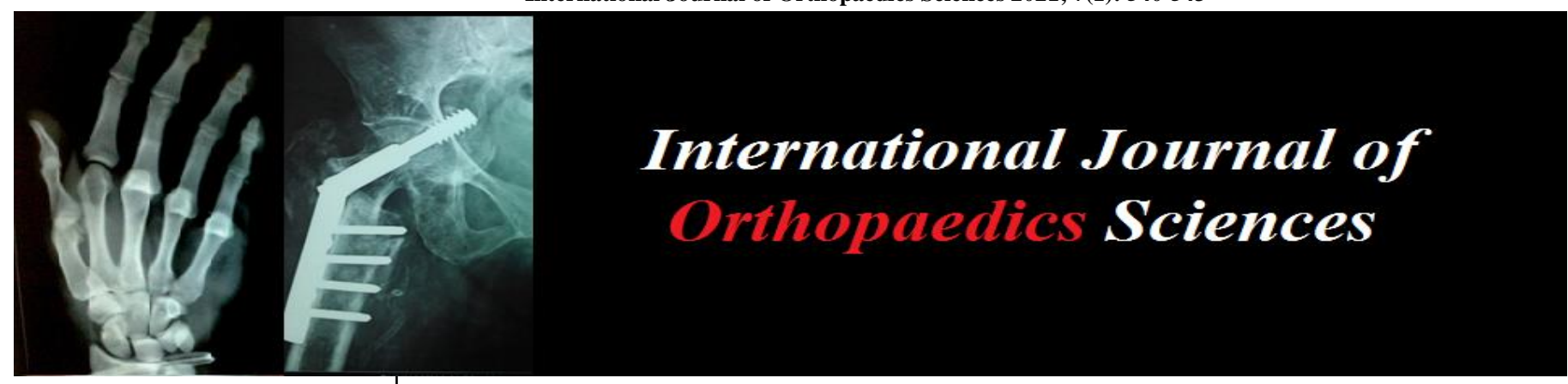

E-ISSN: 2395-1958

P-ISSN: 2706-6630

IJOS 2021; 7(1): 540-543

(C) 2021 IJOS

www.orthopaper.com

Received: 17-11-2020

Accepted: 21-12-2020

Dr. Sabyasachi Bhowmik Associate Professor, Department of Orthopaedic, American International Institute of Medical Sciences, Bedwas, Udaipur, Rajasthan, India

Dr. Mohammad Azmoddin Professor, Department of orthopaedic, American international institute of medica sciences, Bedwas, Udaipur, Rajasthan, India
Corresponding Author: Dr. Mohammad Azmoddin Professor, Department of orthopaedic, American international institute of medical sciences, Bedwas, Udaipur,

Rajasthan, India

\section{Outcome assessment of locking plate fixation for management of displaced olecranon fractures}

\author{
Dr. Sabyasachi Bhowmik and Dr. Mohammad Azmoddin
}

DOI: https://doi.org/10.22271/ortho.2021.v7.i1i.2538

\section{Abstract}

Objectives: To evaluate the outcome of locking plate fixation for management of displaced olecranon fractures.

Materials and Methods: The present observational study was conducted in the department of Orthopedics, American International Institute of Medical Sciences, Udaipur, Rajasthan, India. Total 25 patients, who underwent plate fixation of displaced olecranon fractures, were reviewed. All patients were asked to attend for routine review at two weeks, six weeks, three months and six months after surgery as standard protocol. The clinical results were assessed according to the Broberg and Morrey scale ${ }^{[1]}$, which categorizes results as excellent ( $>95$ points), good (80 to 94 points), fair (60 to 79 points) and poor ( $<60$ points). Analyses of any complications observed in terms of loss of reduction, infection, problems of union, symptomatic prominent hardwire and implant failure.

Results: Mean flexion extension arc of elbow is $116.2^{\circ}$ with mean flexion $125.7^{0}$, mean extension loss $10.2^{\circ}$, mean pronation $78^{\circ}$, mean supination $81.5^{\circ}$ and excellent clinical outcome was reported among $76 \%$ cases as per Broberg and Morrey scale. 22 patients achieved fracture union during a six months follow up period.

Conclusion: Locking plate fixation is an effective treatment option for displaced olecranon fractures with a good functional outcome and a low incidence of complications.

Keywords: olecranon, fracture, plate fixation, ulna

\section{Introduction}

Proximal one third ulna fractures are common adult injuries around the elbow, which comprise a broad spectrum of injuries including olecranon fractures and Monteggia fractures.

The management of complex, comminuted fracture of the olecranon can be extremely difficult, especially when it is combined with fracture of the radial head and/or coronoid process of the ulna or injury of the collateral ligament of the elbow joint ${ }^{[2,3]}$.

The treatment goals are to obtain anatomic reduction that remains stable over time, bone healing and functional recovery. To allow early functional mobilization and to prevent stiffness of the elbow joint, the fracture fixation has to provide secure stability ${ }^{[4,5]}$. Tension band wiring (TBW) is frequently recommended for most simple non-comminuted transverse fractures [6, 9]. Commonly reported complications of TBW include painful hardware prominence, heterotopic ossification, non-union and ulnar nerve neuropraxia ${ }^{[6,10-12]}$. Further rarely described complications are: $\mathrm{K}$-wire penetration into the proximal radio-ulnar joint causing proximal radio-ulnar synostosis ${ }^{[13]}$ and limited pronation and supination ${ }^{[14]}$ ulnar artery injury causing acute ischemia and Volkmann contracture ${ }^{[15,16]}$ transient injury of the anterior interosseous nerve ${ }^{[17]}$ and median nerve injury ${ }^{[18]}$. To overcome these complications of TBW, better stability and early rehabilitation, plate fixation is used for the management of simple olecranon fractures. Also plate fixation is commonly employed for comminuted fractures, because subchondral bony comminution opposite the tension band will cause failure in compression ${ }^{[19,20]}$.

Very few reports have specifically addressed the treatment outcomes of displaced fractures of the olecranon with locking plate. Hence the present study was conducted with the aim to evaluate clinical outcome of the locking plate fixation in cases of displaced olecranon fracture. 


\section{Material \& Methods}

\section{Study Design}

The present observational study was conducted in the department of Orthopedics, American International Institute of Medical Sciences, Udaipur, Rajasthan, India. Total 25 patients who underwent locking compression plate fixation of displaced olecranon fractures were reviewed.

\section{Inclusion criteria}

- Patient who signed the "informed consent" form.

- Patients $\geq 18$ years of age.

- Patients with displaced olecranon fractures less than two weeks duration.

\section{Exclusion criteria}

- Patients $<18$ years of age.

- Patients with additional ipsilateral upper extremity injury.

- Patients who were unfit for surgery.

- Fracture with neurovascular injury.

\section{Methodology}

Pre-operatively the neurovascular status and radiological assessment of the fractured limb were done. The decision of the exact modality of surgery and fixation was made. Surgical antibiotic prophylaxis was given within one hour of surgery. Patients were given general or regional anesthesia. Position of the patient was lateral decubitus with arm over padded bar allowing elbow flexion. Image intensifier was kept for intraoperative imaging if required. Following limb exsanguination, a tourniquet was inflated. A direct posterior midline approach centered on the olecranon and following ulnar ridge was used. For fracture fixation Olecranon locking plate was used. A posterior removable POP slab with the elbow at $90^{\circ}$ flexion was applied in the operating room and maintained for a period of 2 weeks.

Postoperatively antibiotics were given as per hospital protocol. Analgesics and other supportive management were given according to the patient's needs. X-ray of elbow with proximal two third of forearm, anterior posterior and lateral views were taken. The patients were discharged according to the overall well-being of the patient, preferably on the third or fourth day with medications convenient to be taken at home. Range of motion exercises of the shoulder and elbow was begun as soon as possible. All patients were asked to attend for routine review at two weeks, six weeks, three months and six months after surgery. Follow up x-rays were taken at each visit and analyzed for progression of union or any displacement of fracture.

\section{Outcome assessment}

Outcome assessment was done by both clinically and radiologically in all patients at three months and six months. The clinical results were assessed according to the Broberg and Morrey scale ${ }^{1}$, which categorizes results as excellent (> 95 points), good ( 80 to 94 points), fair (60 to 79 points) and poor (< 60 points). Analysis of any complications observed in terms of loss of reduction, infection, problems of union, symptomatic prominent hardwire and implant failure.

\section{Statistical Analysis}

The recorded data was compiled and entered in a spreadsheet computer program (Microsoft Excel 2010) and then exported to data editor page of SPSS version 19 (SPSS Inc., Chicago, Illinois, USA). Descriptive statistics included computation of percentages. The statistical test applied for the analysis was Pearson's chi-square test $\left(\chi^{2}\right)$. The confidence interval and $\mathrm{p}$ value were set at $95 \%$ and $\leq 0.05$ respectively.

\section{Results}

Table 1: Age and gender profile of the study population

\begin{tabular}{|c|c|}
\hline Variables & $\mathbf{N}(\%)$ \\
\hline \multicolumn{2}{|c|}{ Gender } \\
\hline Male & $19(76.0 \%)$ \\
\hline Female & $6(24.0 \%)$ \\
\hline \multicolumn{2}{|c|}{ Age } \\
\hline $28-37$ Years & $6(24.0 \%)$ \\
\hline $38-47$ Years & $11(44.0 \%)$ \\
\hline$>47$ Years & $5(20.0 \%)$ \\
\hline Total & $3(12.0 \%)$ \\
\hline Mean \pm SD & $25(100.0 \%)$ \\
\hline
\end{tabular}

Table 2: distribution patients as per laterality and Mayo classification

\begin{tabular}{|c|c|}
\hline Variables & $\mathbf{N}(\%)$ \\
\hline \multicolumn{2}{|c|}{ Laterality } \\
\hline Right & $9(36.0 \%)$ \\
\hline Left & $16(64.0 \%)$ \\
\hline \multicolumn{2}{|c|}{ Mayo Classification } \\
\hline IIA & $16(64.0 \%)$ \\
\hline IIB & $5(20.0 \%)$ \\
\hline IIIA & $4(16.0 \%)$ \\
\hline Total & $25(100.0 \%)$ \\
\hline
\end{tabular}

Table 3: Range of Motion at 3 months and 6 months follow-up

\begin{tabular}{|c|c|c|}
\hline Variables & 3 months & 6 months \\
\hline Mean flexion extension arc & $92.8^{0}$ & $116.2^{0}$ \\
\hline Mean flexion & $105.2^{0}$ & $125.7^{0}$ \\
\hline Mean extension loss & $11.8^{0}$ & $10.2^{0}$ \\
\hline Mean pronation & $71.6^{\circ}$ & $78^{\circ}$ \\
\hline Mean supination & $72.4^{\circ}$ & $81.5^{\circ}$ \\
\hline Mean pronation supination arc & $141.4^{\circ}$ & $158.5^{\circ}$ \\
\hline
\end{tabular}

Table 4: Clinical outcome according to Broberg and Morrey scale

\begin{tabular}{|c|c|}
\hline Broberg and Morrey scale & $\mathbf{N}(\mathbf{\%})$ \\
\hline Excellent & $19(76.0 \%)$ \\
\hline Good & $4(16.0 \%)$ \\
\hline Fair & $2(8.0 \%)$ \\
\hline Poor & $0(0.0 \%)$ \\
\hline
\end{tabular}

Table 5: distribution of complications

\begin{tabular}{|c|c|}
\hline Complications & $\mathbf{N}(\mathbf{\%})$ \\
\hline Loss of reduction & $0(0.0 \%)$ \\
\hline Infection & $0(0.0 \%)$ \\
\hline Implant failure & $0(0.0 \%)$ \\
\hline Delayed Union & $3(12.0 \%)$ \\
\hline Symptomatic prominent hardwire & $4(16.0 \%)$ \\
\hline
\end{tabular}

A temporary superficial infection limited to around the olecranon, was seen in one patient which was resolved with the use of intravenous antibiotics. 


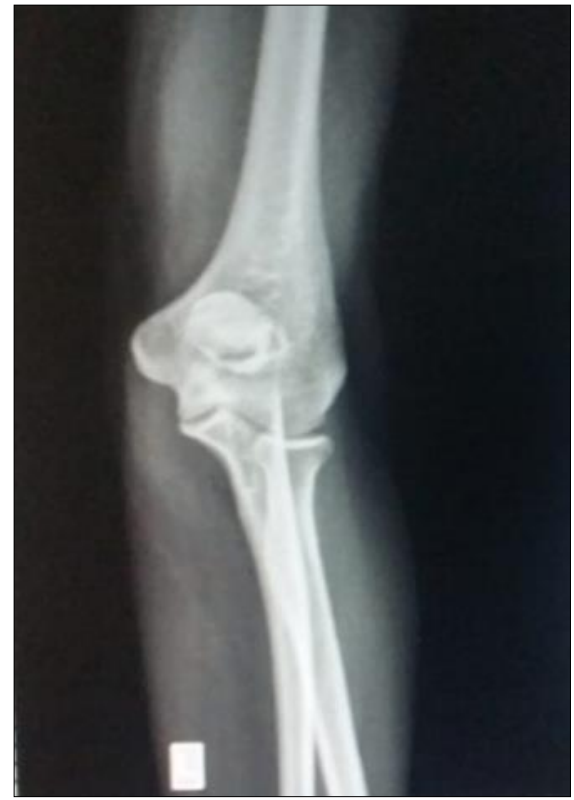

Fig 1: Preoperative X-ray, AP view showing fracture olecranon

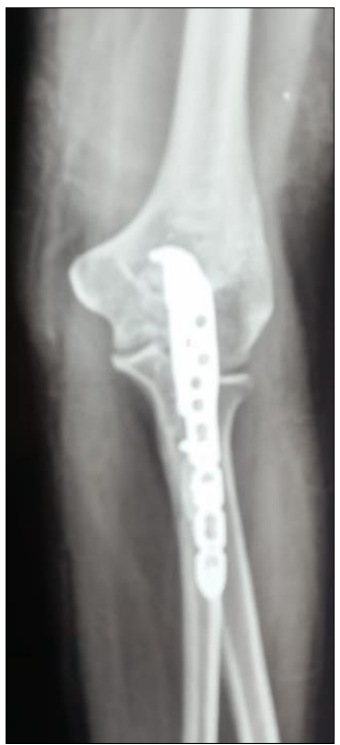

Fig 3: Postoperative $\mathrm{X}$-ray, AP view showing olecranon fracture fixed with locking plate

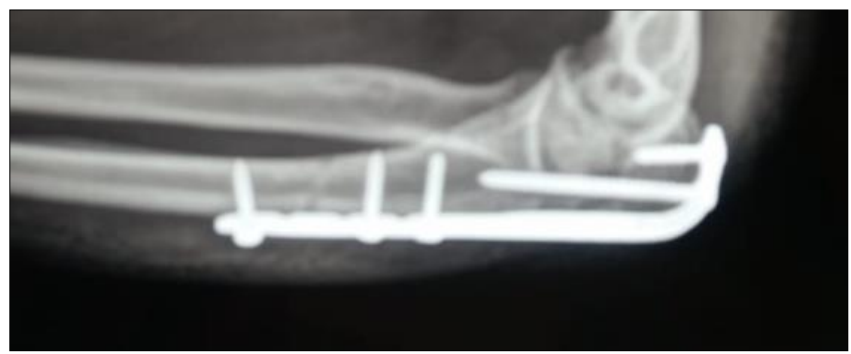

Fig 4: Postoperative $X$-ray, lateral view showing olecranon fracture fixed with locking plate

\section{Discussion}

Open reduction and stable internal fixation with the goal of anatomical reduction of the articular surface is the gold standard for displaced olecranon fracture treatment. Non comminuted olecranon fractures can be treated by tension band wiring or plating. In cases of comminuted fractures of proximal ulna it has many pitfalls like loss of fixation, prominence of hardware, impingement and synostosis.

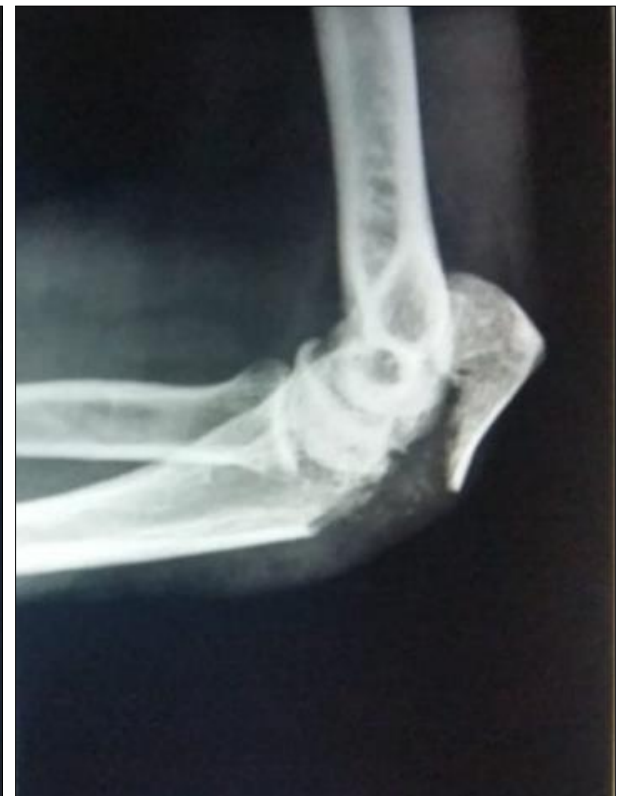

Fig 2: Preoperative X-ray, lateral view showing fracture olecranon

Use of a locking plate avoids these complications and can also be used in comminuted as well as non-comminuted fractures. It also provides structural stability, resists ulnar angulation, and restores ulna length. In addition, plate fixation lowers the risk of fatigue failure caused by extreme bending stresses.

Operative treatment by plating has been shown to provide more predictable alignment and immediate fracture stability, allowing early elbow mobilization.

There are many reports of favorable results using a plate and screws system for stabilizing olecranon fractures. Anderson et al. reported $92 \%$ (22 of 24) good or excellent results after congruent plate fixation of olecranon fracture ${ }^{[21]}$.

In the present study, mean flexion extension arc of elbow is $116.2^{0}$, mean pronation supination arc is $158.5^{\circ}$ and excellent clinical outcome was reported among $76 \%$ cases as per Broberg and Morrey scale. Ring et al. reported 88\% (15 of 17) good or excellent results after plate fixation of transolecranon fracture dislocations, with a mean flexion of $127^{\circ} .22$ Mortazavi et al. treated eight patients with trans-olecranon fracture dislocations; all seven treated with plating had a good or excellent result ${ }^{[23]}$

To our knowledge, few reports have specifically addressed the treatment outcomes of comminuted fractures of the olecranon. Ikeda et al. treated ten patients with comminuted fractures of the olecranon by multiple tension-band wiring and a graft from the iliac crest. They reported that $100 \%$ of their patients had an excellent or good result, with a mean flexion of $135^{\circ}$ with a mean flexion contracture of 15 degrees [24]

Very few complications are found in this study with plate fixation - symptomatic prominent hardwire (16.0\%) was commonest followed by delayed Union (12.0\%). In Schliemann B et al. study, implant irritation (with subsequent removal) was the most common complication [7 in locking compression plate (LCP) groups, and 12 in TBW groups] ${ }^{[25]}$. At the final follow up of six months we did not advise any patient of implant removal, as we feel that this period is too early to implant removal. There are some limitations in this study. Follow up period is relatively short and sample size is small. Further study with prolonged follow up and larger sample size will eliminate these problems. 


\section{Conclusion}

Management of displaced olecranon fracture fixed with locking plate, shows excellent to good functional outcome and a low incidence of complications. Locking plate fixation gives better stability which helps in early rehabilitation in displaced olecranon fracture.

\section{References}

1. Broberg MA, Morrey BF. Results of treatment of fracture-dislocations of the elbow. Clin Orthop Relat Res 1987;216:109-119.

2. Hak DJ, Golladay GJ. Olecranon fractures: treatment options. J Am Acad Orthop Surg 2000;8:266-275.

3. Quintero J, Vareck T. Olecranon, radial head, and complex elbow injuries. In: Ruedi TP, Buckley RE, Moran CG, eds. AO principles of fracture management, vol 2: Specific fractures, 2nd edn. Stuttgart: Thieme 2007, 626-41.

4. Newman SDS, Mauffrey C, Krikler S. Olecranon fractures. Injury 2009;40:575-581.

5. Sahajpal D, Wright TW. Proximal ulna fractures. J Hand Surg Am 2009;34:357-362.

6. Holdsworth BJ, Mossad M. Elbow function following tension band fixation of displaced fractures of the olecranon. Injury 1984;16:182-87.

7. Rowland SA, Burkhart SS. Tension band wiring of olecranon fractures: a modification of the AO technique. Clin Orthop 1992;227:238-42.

8. Sadri H, Stern R, Singh M, Linke B, Hoffmeyer P, Schwieger K. Transverse fractures of the olecranon: A biomechanical comparison of three fixation techniques. Arch Orthop Trauma Surg 2011;131:131-138.

9. Molloy S, Jasper Le, Elliott DS, Brumback RJ, Belkoff $\mathrm{Sm}$. Biomechanical evaluation of intramedullary nail versus tension band fixation for transverse olecranon fractures. J Orthop Trauma 2004;18:170-174.

10. Macko D, Szabo RM. Complications of tension-band wiring of olecranon fractures. J Bone Joint Surg Am 1985;67:1396-1401.

11. Helm RH, Hornby R, Miller SW. The complications of surgical treatment of displaced fractures of the olecranon. Injury 1987;18:48-50.

12. Koslowsky TC, Mader K, Dargel J, Schadt R, Koebke J, Pen-nig D. Olecranon fracture fixation with a new implant: biomechanical and clinical considerations. Injury 2009;40:618-624.

13. De Carli P, Gallucci GL, Donndorff Ag, Boretto Jg, Alfie VA. Proximal radio-ulnar synostosis and nonunion after olecranon fracture tension-band wiring: a case report. J Shoulder elbow Surg 2009;18:e40-e44.

14. Candal-Couto JJ, Williams Jr, Sanderson PL. Impaired forearm rotation after tension-band-wiring fixation of olecranon fractures: evaluation of the transcortical Kwire technique. J Orthop Trauma 2005;19:480-482.

15. Rompen JC, Vos GA, Verheyen CC. Acute ischemia of the hand seven months after tension-band wiring of the olecranon. J Shoulder elbow Surg 2010;19:e9-e11.

16. Lee SH, Han SB, Jeong WK, Park JH, Park Sy, Patil S. Ulnar artery pseudoaneurysm after tension band wiring of an olecranon fracture resulting in Volkmann's ischemic contracture: a case report. J Shoulder elbow Surg 2010;19:e6-e8.

17. Parker Jr, Conroy J, Campbell DA. Anterior interosseus nerve injury following tension band wiring of the olecranon. Injury 2005;36:1252-1253.
18. Thumroj E, Jianmongkol S, Thammaroj J. Median nerve palsy after operative treatment of olecranon fracture. J med Assoc Thai 2005;88:1434-1437.

19. Tan SL, Balogh ZJ. Inidications and limitations of locked plating. Injury 2009;40:683-691.

20. Siebenlist S, Torsiglieri T, Kraus T, Burghardt RD, Stöckle U et al. Comminuted fractures of the proximal ulna-Preliminary results with anatomically preshaped locking compression plate (LCP) system. Injury 2010;41(12):1306-11.

21. Anderson ML, Larson AN, Merten SM et al. Congruent elbow plate fixation of olecranon fractures. J Orthop Trauma 2007;21:386-93.

22. Ring D, Jupiter JB, Sanders RW et al. Transolecranon fracture-dislocation of the elbow. J Orthop Trauma 1997;11:545-550.

23. Mortazavi SM, Asadollahi S, Tahririan MA. Functional outcome following treatment of transolecranon fracturedislocation of the elbow. Injury 2006;37:284-288.

24. Ikeda M, Fukushima Y, Kobayashi Y et al. Comminuted fractures of the olecranon. Management by bone graft from the iliac crest and multiple tensionband wiring. J Bone Joint Surg Br 2001;83:805-808.

25. Schliemann B, Raschke MJ, Groene P, Weimann A, Wähnert D, Lenschow $\mathrm{S}$ et al. Comparison of tension band wiring and precontoured locking compression plate fixation in mayo type IIA olecranon fractures. Acta Orthop Belg 2014;80(1):106-11. 